\title{
Assessing the Relationship Between Drive for Thinness and Taste-Shape Correspondences
}

\author{
Yumi Hamamoto ${ }^{1,2, *}$, Kosuke Motoki ${ }^{1,3}$ and Motoaki Sugiura ${ }^{1,4}$ \\ ${ }^{1}$ Institute of Development, Aging and Cancer, Tohoku University, Sendai, Japan \\ ${ }^{2}$ Japan Society for the Promotion of Science, Tokyo, Japan \\ ${ }^{3}$ Department of Food Management, School of Food, Agricultural and Environmental \\ Sciences, Miyagi University, Sendai, Japan \\ ${ }^{4}$ International Research Institute of Disaster Science, Tohoku University, Sendai, Japan
}

Received 31 January 2020; accepted 13 June 2020

\begin{abstract}
Eating disorder tendencies are psychological characteristics that are prevalent in healthy young females and are known to be among the risk factors for eating disorders such as anorexia and bulimia nervosa. People with greater eating disorder tendencies strongly associate sweet and fatty foods with weight gain and strictly avoid consuming such foods. However, little is known about how eating disorder tendencies influence the association between taste and body shape impression. Research on crossmodal correspondences suggests that people preferentially associate sweet tastes with round shapes, and individual differences affect the degree of such associations. This study investigates how the degree of taste-shape matching is related to eating disorder tendencies with a preliminary investigation of what mediates this relationship. Two experiments were conducted: in Experiment 1, healthy participants rated the degree of association between basic taste words (sweet/sour/salty/bitter) and roundness of shape and subsequently completed questionnaires addressing eating disorder tendencies. In Experiment 2, participants answered additional questionnaires addressing obsessiveness, dichotomous thinking, and self-esteem. The results of Experiment 1 indicated a positive correlation between drive for thinness, which is one indicator of an eating disorder tendency, and the degree of matching sweetness to round shape. Experiment 2 replicated the results of Experiment 1 and revealed the mediating effect of obsessiveness. These findings suggest a relationship between individual differences in taste-shape matching and eating disorder tendency and the preliminary mediating role of obsessiveness. The present study provides new insight into the role of sweet-round matching in eating disorder tendencies and the associated psychological mechanisms.
\end{abstract}

\section{Keywords}

eating disorder, crossmodal correspondences, sweet, round shapes, obsessive-compulsive symptoms

\footnotetext{
* To whom correspondence should be addressed. E-mail: yumi.hamamoto.q2@dc.tohoku.ac.jp
} 


\section{Introduction}

\subsection{Perceptual Bias Related to Eating Disorder Tendencies}

Anorexia nervosa and bulimia nervosa are psychiatric disorders categorized as feeding and eating disorders. These disorders are characterized by maladaptive eating behaviors that originate from individuals' concerns regarding their weight and body shape (American Psychiatric Association, 2013). People who suffer from these eating disorders avoid consuming high-calorie foods in an effort to prevent weight gain, and this behavior sometimes persists after weight recovery (Foerde et al., 2015; Gwirtsman et al., 1989; Jáuregui Lobera and Bolaños Ríos, 2009). It has been suggested that people with greater eating disorder tendencies, including nonclinical populations, perceive taste and shape differently to individuals with lower eating disorder tendencies. People with greater eating disorder tendencies show, in particular, different body shape perceptions. They are unable to estimate their body size accurately, with a tendency toward overestimation (Gardner and Bokenkamp, 1996; Hamamoto et al., 2019; Mohr et al., 2010; Smeets et al., 1999). Furthermore, it has been demonstrated that taste perception in patients with eating disorders differs from that of healthy individuals (Chao et al., 2019; Dazzi et al., 2013; Kinnaird et al., 2018; Oberndorfer et al., 2013; Wagner et al., 2015). Several studies have focused on the perception of sweet taste because patients with eating disorders often avoid sweet foods. Participants who had recovered from eating disorders exhibited different patterns of sensitization to repetitive sweet stimuli, as compared to those exhibited by healthy people (Wagner et al., 2015). This difference in sensitization patterns was supported by a neuroimaging study that observed varying brain activity in the insula, which contains the primary gustatory cortex (Pritchard et al., 1999; Oberndorfer et al., 2013). Although considerable research has been conducted in this field, these differences in shape and taste perception have been investigated independently. Given that individuals with greater eating disorder tendencies avoid sweet foods to prevent weight gain and may strongly associate their body shape with sweet tastes, eating disorder tendencies may relate to the associations between shape and taste perceptions.

\subsection{Taste-Shape Matching: Crossmodal Correspondences}

Studies on crossmodal correspondences have demonstrated associations between shape and taste. The term crossmodal correspondences refers to the phenomenon whereby people associate features in one sensory modality with features in other modalities in a consistent manner (Spence, 2011). Earlier research has documented correspondences between shape and taste (Motoki et al., 2019a; Spence and Deroy, 2013; Velasco et al., 2016a). Sweet taste is reliably matched with rounded shapes, while sour taste corresponds significantly 
to angularity (Ngo et al., 2013). Sweet taste is also matched with symmetric rather than asymmetric shapes, while sour taste is associated with no such preference (Turoman et al., 2018). The association between sweet taste and roundness is not limited to simple shapes: people match sweet tastes to round faces (Motoki et al., 2019a). Furthermore, it has been suggested that there are individual differences in the degree of matching between taste and shape roundness (Velasco et al., 2015). For example, sweet tastes were generally associated with round shapes, but the degree of this sweet-round matching varied across participants (Velasco et al., 2015). This earlier finding suggests that personality traits underlie the association between shape and taste. Thus, we hypothesize that eating disorder tendencies will also influence taste-shape matching.

\subsection{Hypothesis Development}

We hypothesized that taste-shape matching, particularly sweet-round matching (i.e., the degree of association between sweet taste and shape roundness), is related to eating disorder tendencies. Shafran et al. (1999) revealed that individuals with greater eating disorder tendencies exhibited specific cognitive distortion. Eating disorder tendency was associated with the belief that simply thinking about eating a forbidden food (sweet foods) could lead to weight gain (thought-shape fusion: Shafran et al., 1999). Taste-shape matching might explain this cognitive distortion. Patients believe that they will gain weight just by thinking about consuming high-calorie foods, perhaps because obesity (round shape) is strongly associated with high-calorie foods (sweet tastes).

H1: People who have greater eating disorder tendencies will strongly associate sweet tastes with round shapes.

As a preliminary exploration study, it was also hypothesized that specific personality traits would mediate the relationship between eating disorder tendencies and sweet-round matching. The mediating effects of the following personality traits were investigated: obsessiveness, dichotomous thinking, and low self-esteem (Byrne et al., 2008; Legenbauer et al., 2011; Oshio and Meshkova, 2012; Palascha et al., 2015; Shafran et al., 1999). It is suggested that obsessiveness is related to the association between thought-shape fusion and eating disorder tendencies (Coelho et al., 2012; Radomsky et al., 2002; Shafran et al., 1999). Consequently, we expected that obsessiveness would mediate the association between sweet-round matching and eating disorder tendencies. Dichotomous thinking is a black or white cognitive thinking style. This style affects individuals' attitudes toward food, their own body image, and their food intake (Byrne et al., 2008; Oshio and Meshkova, 2012; Palascha et $a l ., 2015)$. It was hypothesized that dichotomous thinking may cause an extreme association between sweet tastes and round shapes. Moreover, patients with eating disorders often have low self-esteem and judge their self-worth 
primarily according to their body weight and shape (American Psychiatric Association, 2013; Legenbauer et al., 2011). This low self-esteem may engender attentional biases toward weight, body checking, food, and calories (Aspen et $a l ., 2013)$. As such, individuals who have lower self-esteem may also perceive stronger associations between sweet tastes and body shape.

\subsection{The Present Study}

This study aimed to clarify the relationship between individual differences in taste-shape matching and eating disorder tendency. Additionally, potential personality traits mediating this relationship were investigated. To this end, two experiments were conducted. The purpose of Experiment 1 was to investigate, exploratorily, relationships between taste-shape matching and eating disorder tendencies. The main purpose of Experiment 2 was to replicate the results of Experiment 1, and the mediator of the relationship between taste-shape matching and eating disorder tendency was preliminarily investigated. Across both experiments, the healthy participants matched basic taste words (sweet/sour/salty/bitter) with angular or round shapes. They subsequently completed questionnaires relating to personality traits, such as eating disorder tendencies.

\section{Material and Methods}

\subsection{Recruiting Procedure and Ethical Approval}

We recruited participants via Lancers (https://www.lancers.jp/) and distributed the survey via Qualtrics (https://www.qualtrics.com/jp/). This study was approved by the Ethics Committee of Tohoku University Graduate School of Medicine, Japan, and was conducted in accordance with the Declaration of Helsinki.

\subsection{Taste-Shape Matching Task}

In both Experiments 1 and 2, the degree of taste-shape matching was assessed. Participants were shown each basic taste word (sweet/sour/salty/bitter) and were required to indicate the extent to which they associate each taste word with the shape symbolic scales anchored with angular (left-hand side) or round (right-hand side) shapes. Each pair of shapes was on a slider-controlled visual analog scale (VAS) ranging from 0 (most likely associated with angular shape) to 100 (most likely associated with round shape). A similar exercise was used in an earlier study on crossmodal correspondences between taste and shape (Velasco et al., 2015). Four types of shape pairs were used (Velasco et al., 2015; Fig. 1); thus, the number of trials was 16 (i.e., 4 shape pairs $\times 4$ taste words). 
Sweet

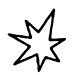

Salty
Sour

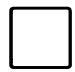

Bitter

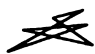

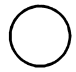

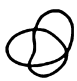

Figure 1. Stimuli used in the taste-shape matching task. Participants indicated the extent to which they associate taste with shape using the visual analog scale (VAS) (the scale ranged from 0 to 100, with 0 on the left and 100 on the right). When a participant associated sweet taste with round shape, they would move the VAS to the right. We investigated all combinations of taste words and shape pairs; thus, participants underwent 16 trials (4 shape pairs $\times 4$ taste words).

\section{Experiment 1}

\subsection{Participants}

We recruited 295 people for Experiment 1; 133 people were excluded according to the following exclusion criteria: failure to respond to all questionnaire items and failure to answer two filler questions. Many participants failed to answer all questionnaire items because the questionnaire items were not set in forced-choice format and no reminder was implemented to notify participants that they had forgotten to select items. Finally, data from 162 participants were analyzed (mean age $\pm \mathrm{SD}=40.6 \pm 10.4$ years old, mean body mass index \pm $\mathrm{SD}=21.9 \pm 3.7)$.

\subsection{Questionnaire}

Participants answered the Japanese version of Eating Disorder Inventory 2 (EDI2; Shimura et al., 1994) after the taste-shape matching task to measure their eating disorder tendencies. This is a self-report questionnaire comprising 11 subscales relating to eating disorder tendency, including drive for thinness, bulimia, body dissatisfaction, ineffectiveness, perfectionism, interpersonal distrust, interoceptive awareness, maturity fears, asceticism, impulse regulation, and social insecurity. Participants answered each question according to a six-point Likert scale where $1=$ never and $6=$ always. The modified scoring system (e.g., modifying 1-3 scores into 0 , and modifying 4-6 scores into 1-3) that is usually used to evaluate the severity of eating disorder symptoms was not applied, because raw scoring (e.g., a score of ' 1 ' where participants rated ' 1 ') is considered better for evaluating eating disorder tendencies in healthy individuals (Schoemaker et al., 1994). 

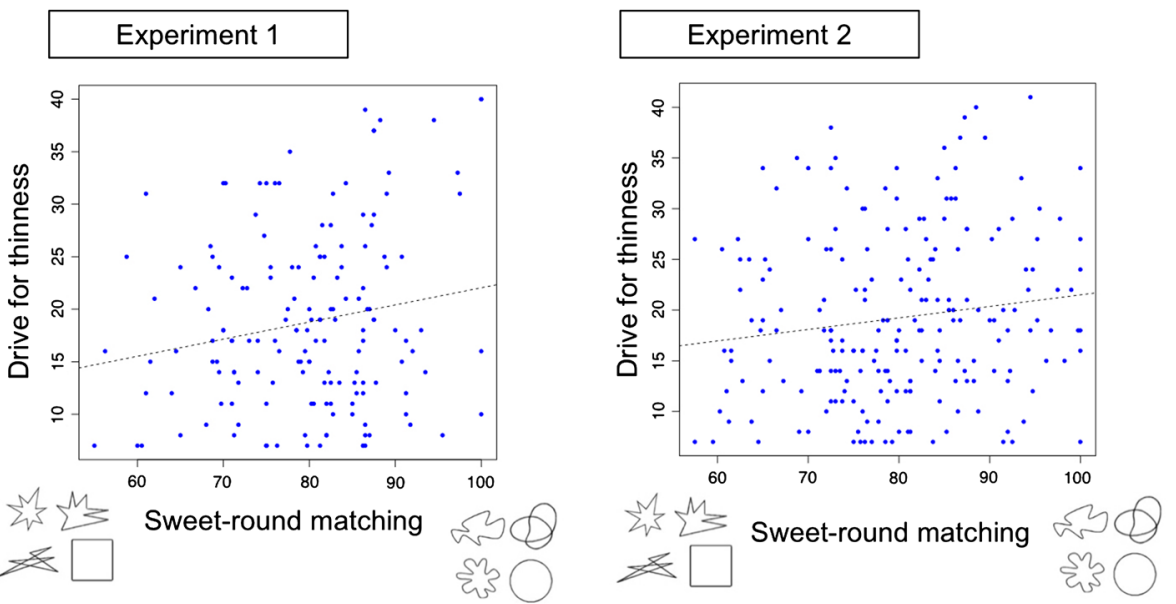

Figure 2. Scatter plot of the degree of sweet-round matching and drive for thinness. The left graph shows the correlation in Experiment $1(r=0.179, p=0.023)$, and the right graph shows the correlation in Experiment $2(r=0.141, p=0.041)$. The sweet-round matching score was distributed from 0 to 100 , where 0 indicates that participants associated sweet tastes with angular shapes (i.e., shapes on the left side of the horizontal axis) and 100 means that participants associated sweet tastes with round shapes (i.e., shapes on the right side of the horizontal axis).

\subsection{Statistical Analysis}

The degree of taste-shape matching was calculated first. Participants underwent four taste-shape matching task trials for each taste, and we scored the degree of taste-shape matching as the average of these four trial scores.

Next a two-tailed simple correlation analysis was conducted to investigate the relationship between taste-shape matching and eating disorder tendency. There were four tastes (sweet/sour/salty/bitter) and 11 eating disorder tendencies from EDI2, and all correlations between the degree of taste-shape matching and eating disorder tendencies (i.e., 4 taste-shape matching $\times 11$ subscales from EDI2) were analyzed. Pearson's correlation coefficients were calculated using R version 3.3.3 (https://www.R-project.org/), and statistical significance was set at $p<0.05$.

\subsection{Results: Experiment 1}

The analysis demonstrated that the degree of sweet-round matching was positively correlated with drive for thinness $(r=0.179, p=0.023$; Fig. 2, left). Positive correlations between the degree of sour-round matching and ineffectiveness $(r=0.257, p<0.001)$, interpersonal distress $(r=0.195$, $p=0.013)$, and social insecurity $(r=0.184, p=0.019)$ were also observed. The analysis also revealed negative correlations between degree of bitterround association and asceticism $(r=-0.223, p=0.004)$ and perfectionism 
Table 1.

Correlation coefficients between the degree of taste-shape matching and eating disorder tendencies in Experiment 1. In Experiment 1, the correlations between the degree of taste-shape matching (i.e., matching between roundness and each taste) and the subscales from EDI2 were investigated. Positive correlation means that participants with greater eating disorder tendencies more strongly associated the relevant tastes with round shape. By contrast, negative correlation means that a greater eating disorder tendency would cause a stronger association between the relevant tastes and angular shape

\begin{tabular}{lcccc}
\hline & Sweet-round & Sour-round & Salty-round & Bitter-round \\
\hline Asceticism & 0.054 & 0.082 & 0.095 & $-0.223^{*}$ \\
Bulimia & 0.103 & 0.008 & 0.050 & -0.039 \\
Body dissatisfaction & 0.001 & 0.003 & 0.048 & -0.001 \\
Drive for thinness & $0.179^{*}$ & -0.112 & -0.066 & -0.106 \\
Ineffectiveness & -0.111 & $0.257^{*}$ & 0.042 & 0.012 \\
Interoceptive awareness & 0.033 & 0.114 & 0.088 & -0.008 \\
Interpersonal distrust & -0.066 & $0.195^{*}$ & 0.150 & 0.029 \\
Impulse regulation & 0.034 & 0.022 & 0.127 & 0.009 \\
Maturity fear & -0.083 & 0.091 & -0.052 & -0.078 \\
Perfectionism & 0.082 & -0.039 & -0.117 & $-0.168^{*}$ \\
Social insecurity & -0.037 & $0.184^{*}$ & 0.119 & -0.001 \\
\hline
\end{tabular}

$*, p<0.05$.

( $r=-0.168, p=0.032$ ). No further significant correlations were observed, and all correlations, including those that were non-significant, are presented in Table 1.

\subsection{Summary and Short Discussion: Experiment 1}

The correlation between the degree of sweet-round matching and eating disorder tendencies was investigated. As expected, a positive correlation between the degree of sweet-round matching and drive for thinness was found. Drive for thinness is among the body image problems that are characteristic of eating disorders. We hypothesized that the relationship between sweet-round matching and eating disorder tendencies has its origin in the consumption of high-calorie foods and subsequent weight gain; this result tends to support our hypothesis. However, significance was lost after correcting for multiple comparisons where Experiment 1 was exploratory, such that all possible correlations were analyzed. Experiment 2 was performed to confirm the correlation between sweet-round matching and drive for thinness.

Several positive and negative correlations between taste-shape matching and eating disorder tendencies for other shapes were also found, although these correlations were not pertinent to our hypothesis. 


\section{Experiment 2}

\subsection{Participants}

Based on the results of Experiment 1, power analysis was conducted to determine the appropriate sample size for Experiment 2. We used G*Power 3.1 (Faul et al., 2009) and set the effect size at 0.179, which was the result obtained from Experiment 1. Effect size $(f=0.179)$, alpha level $(a=0.05)$, and power $(1-b=0.8)$ were input into $\mathrm{G}^{*}$ Power. The required sample size was 279 . Thus, 307 people were recruited, and 96 were excluded in accordance with the same exclusion criteria as in Experiment 1. The drop-out rate was high because, again, the questionnaire items were not set in forced-choice format and no reminder was implemented to notify participants that they had forgotten to select items. Data from 211 participants were finally analyzed for Experiment 2 (mean age $\pm \mathrm{SD}=40.1 \pm 10.1$ years old, mean body mass in$\operatorname{dex} \pm \mathrm{SD}=22.1 \pm 3.8$ ). Power analysis showed that the study had sufficient power, despite the smaller than intended sample size (power $=0.75$ ).

\subsection{Questionnaire}

In addition to EDI2, we adopted several questionnaires aimed at measuring participants' personality traits that may mediate between eating disorder tendency and taste-shape matching. These questionnaires included the YaleBrown Obsessive-Compulsive Scale (Y-BOCS), the Rosenberg Self-Esteem Scale (RSES), and the Dichotomous Thinking Inventory (DTI). Participants answered the Japanese version of the Y-BOCS (Hamagaki et al., 1999), which is widely used to measure obsessive-compulsive traits. It comprises two sections: questions about obsessive thoughts and questions about compulsive behaviors. All questions were summed up to evaluate participants' obsessiveness. Participants also completed the Japanese version of DTI (Oshio, 2009), which assesses the tendency toward a dichotomous thinking style. Finally, participants answered the Japanese version of RSES (Mimura and Griffiths, 2007), which evaluates participants' self-esteem. It is widely known that people with eating disorders tend to have low self-esteem and that low self-esteem is a key risk factor in the development of eating disorders (American Psychiatric Association, 2013).

Although it was not the focus of our study, some questions relating to sweet taste and round shape were included in the questionnaire for the purpose of detecting potential mediators. Participants answered questions relating to frequency and emotion regarding sweet tastes and shape. Earlier research suggested that frequent experience of two different stimulus modality sets (e.g., sweet tastes and round shape) caused crossmodal correspondences (Ernst, 2007; Parise et al., 2014; Spence, 2011). Furthermore, emotion category (e.g., both sweet tastes and round shape are associated with positive emotion) is also 
associated with crossmodal correspondences (Motoki et al., 2020; Spence, 2019; Velasco et al., 2015). Thus, participants answered questions relating to frequency (i.e., how often they hear/see information pertaining to the relationship between sweet foods and round body shape) and emotion (i.e., how much they associate sweet food/round shape with negative feelings; how much they associate sweet food/round shape with positive feelings, and how much they associate sweet food/round shape with saliency). Questions pertaining to the relationship between body shape/weight and taste/shape were also included: how much do you associate sweet foods with your own body shape; how much do you associate sweet food/round shape with obesity; how much do you associate sweet food/round shape with weight; how fearful are you that your body shape will change as a result of eating sweet foods; and how much do you perceive yourself as gaining weight by eating sweet foods?

\subsection{Statistical Analysis}

A two-tailed simple correlation analysis was performed to confirm the results of Experiment 1. A further two-tailed simple correlation analysis was conducted to investigate the correlation between taste-shape matching, eating disorder tendency, and personality traits (obsessiveness, dichotomous thinking, self-esteem, frequency, and emotions relating to sweet taste and round shape).

Regarding the correlation between sweet-round matching and drive for thinness, multiple comparison correction was not conducted. Previous studies have suggested that if there is a primary outcome, multiple comparison correction is not needed (Althouse, 2016; Feise, 2002; Li et al., 2017). Experiment 2 had the primary outcome (the correlation between sweet-round matching and drive for thinness), which was set based on the result from Experiment 1. Thus, statistical significance regarding the correlation between sweet-round matching and drive for thinness was set at $p<0.05$ without multiple comparison. However, regarding potential mediating factors, Experiment 2 was an exploratory study and included no a-priori hypothesis. Thus, multiple comparison correction was conducted in the analysis of the correlation regarding potential mediating factors (i.e., obsessiveness, dichotomous thinking, selfesteem, frequency of exposure, emotional reaction, and body image issues relating to sweet taste/round shape). The false discovery rate (FDR) was controlled based on previous research (Benjamini and Hochberg, 1995). For this analysis, statistical significance was set at the FDR-corrected $p$-value $<0.05$.

In Experiment 2, it was hypothesized that the relationship between tasteshape matching and eating disorder tendency is mediated by personality traits (obsessiveness, dichotomous thinking, self-esteem, frequency, emotion, and body-related issues regarding sweet taste/round shape). To this end, mediation analysis was conducted using the PROCESS macro for SPSS (Hayes, 2013). 
Table 2.

Correlation coefficients between the degree of taste-shape matching and target personality traits in Experiment 2. In Experiment 2, the correlations between taste-shape matching and other personality traits were investigated in addition to drive for thinness from EDI2. Obsessiveness, dichotomous thinking and self-esteem were respectively evaluated by Y-BOCS, DTI, and RSES. Positive correlation means that participants with a greater eating disorder tendency more strongly associated the relevant taste with round shape. By contrast, a negative correlation means that a greater eating disorder tendency causes a stronger association between relevant taste and angular shape

\begin{tabular}{lcccc}
\hline & Sweet-round & Sour-round & Salty-round & Bitter-round \\
\hline Drive for thinness & $0.141^{\mathrm{a}}$ & -0.014 & -0.029 & -0.066 \\
Obsessiveness & $0.166^{\mathrm{b}}$ & 0.025 & -0.010 & -0.050 \\
Dichotomous thinking & 0.073 & -0.066 & 0.064 & 0.024 \\
Self-esteem & -0.076 & 0.058 & 0.065 & -0.038 \\
\hline
\end{tabular}

a , $p<0.05$; $^{\mathrm{b}}$, FDR-corrected $p$-value $<0.05$.

The significance of the indirect effect was evaluated using the bias-correlated bootstrapping method (Efron and Tibshirani, 1993; MacKinnon et al., 2004). Bootstrapping analysis using 5000 bootstrap samples was performed. According to previous studies (Efron and Tibshirani, 1993; MacKinnon et al., 2004; Preacher and Hayes, 2004), indirect effect was regarded as significantly different from zero when the $95 \%$ bias-corrected confidence intervals did not include zero.

\subsection{Results: Experiment 2}

\subsubsection{Correlation Between the Degree of Taste-Shape Matching and Personality Traits}

We successfully replicated the result that indicated a positive correlation between sweet-round matching and drive for thinness $(r=0.141, p=0.041$, Fig. 2, right). In Experiment 2, positive correlations between sweet-round matching and other eating disorder tendencies (bulimia, body image dissatisfaction, interoceptive awareness, and impulse regulation; Supplementary Table 1) were observed, although Experiment 1's results did not confirm such a correlation. Sweet-round matching was also positively correlated with obsessiveness $(r=0.166$, FDR-corrected $p$-value $=0.032$ ). These correlations between the degree of sweet-round matching and target personality traits (i.e., drive for thinness, obsessiveness, dichotomous thinking, and self-esteem) are detailed in Table 2, and all results are presented in Supplementary Table 1. 


\section{Table 3.}

Correlation coefficient between drive for thinness and other personality traits in Experiment 2. To determine a mediation factor, we investigated the correlation between drive for thinness and personality traits, namely obsessiveness, dichotomous thinking, and self-esteem. Drive for thinness was evaluated using EDI2. Obsessiveness, dichotomous thinking and self-esteem were respectively evaluated by Y-BOCS, DTI, and RSES

\begin{tabular}{lc}
\hline & Drive for thinness \\
\hline Obsessiveness & $0.216^{*}$ \\
Dichotomous thinking & 0.130 \\
Self-esteem & -0.172 \\
\hline
\end{tabular}

*, FDR-corrected $p$-value $<0.05$.

\subsubsection{Correlation Between Eating Disorder Tendency and Other Personality Traits}

Based on the results of Experiment 1, the correlations between various factors and drive for thinness were investigated (Table 3). Drive for thinness was positively correlated with obsessiveness $(r=0.216$, FDR-corrected $p$-value $=0.005)$ and negatively correlated with self-esteem $(r=-0.172$, FDR-corrected $p$-value $=0.02$ ) .

Although it is not our main hypothesis, significant correlations between drive for thinness and questionnaire scores related to sweet taste and round shape were also observed. Drive for thinness was positively correlated with negative feelings toward sweet taste/round shape $(r=0.181$, FDR-corrected $p$-value $=0.019)$. Additionally, participants who had a higher drive for thinness more strongly associated sweet foods/round shape with obesity $(r=$ 0.179 , FDR-corrected $p$-value $=0.017)$, weight $(r=0.152, p=0.027)$ and $\operatorname{diet}(r=0.148$, FDR-corrected $p$-value $=0.04)$. Participants having a higher drive for thinness also reported stronger association between sweet foods and their own body shape $(r=0.623$, FDR-corrected $p$-value $<0.001)$, stronger fear of changing body shape by eating sweet foods $(r=0.745$, FDR-corrected $p$-value $<0.001)$, and stronger beliefs that they would gain weight by eating sweet foods $(r=0.470$, FDR-corrected $p$-value $<0.001)$. No further significant correlations were observed. All correlations, including other EDI2 subscales, are presented in Supplementary Table 2.

\subsubsection{Mediation Analysis}

Our analysis showed that obsessiveness was the only factor that significantly correlated with both sweet-round matching and drive for thinness. Therefore, mediation analysis was conducted to examine whether obsessiveness mediated the effect of drive for thinness on sweet-round matching. The indirect 


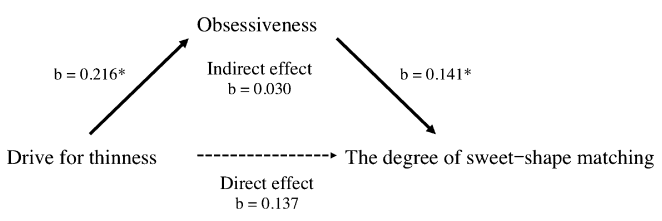

Figure 3. Model to predict the effect of drive for thinness on the degree of taste-shape matching mediated by obsessiveness in Experiment 2. Although the effect was small, obsessiveness had a significant mediating effect on drive for thinness and the degree of taste-shape matching. Standardized coefficients $(b)$ are displayed. Asterisks indicate significant paths $\left(p<0.05^{*}\right)$.

effect was estimated using unstandardized regression coefficient by setting drive for thinness as the independent variable $(X)$, sweet-round matching as the outcome variable $(Y)$, and obsessiveness as the mediator variable $(M)$. The bootstrap estimates were positive and the $95 \%$ bias-corrected confidence intervals did not include zero. The total indirect effect was $0.038, \mathrm{SE}=0.022$, CI [0.002, 0.087] (Fig. 3).

\subsection{Summary and Short Discussion: Experiment 2}

Experiment 2 replicated the results of Experiment 1 in terms of the positive correlation between the degree of sweet-round matching and drive for thinness. It was also found that obsessiveness mediated this relationship. No other factors (e.g., self-esteem) were correlated with both sweet-round matching and drive for thinness. Therefore, we could not investigate the mediating role of other mediation factors. Other correlations between eating disorder tendencies and taste-shape matching observed in Experiment 1 were not replicated.

\section{Discussion}

\subsection{Summary of Findings}

This study examined relationships between the magnitude of taste-shape matching and the degree of eating disorder tendency in healthy adults. The results of Experiment 1 demonstrated that the degree to which individuals match sweet taste to round shape is positively correlated with drive for thinness. The results of Experiment 2 replicated the findings of Experiment 1 and observed the potential mediating role of obsessiveness in the relationship between sweet-round matching and drive for thinness. Taken together, these findings suggest that individual differences in taste-shape perception relate to a subset of eating disorder tendencies (i.e., drive for thinness) and that obsessiveness mediates this relationship. These findings offer a novel explanation for the psychological mechanisms of eating disorder tendencies. 


\subsection{Theoretical Contributions: the Role of Sweet-Round Matching in Eating} Disorders

The correlation between sweet-round matching and drive for thinness suggests that eating disorder tendencies relate to individual differences in sensory matching. Previous studies have reported that people with greater body image concerns - an indicator of eating disorder tendencies - tend to believe that they will gain weight by simply thinking about eating high-calorie foods (Coelho et al., 2012; Radomsky et al., 2002; Shafran et al., 1999). Based on these previous studies, it was hypothesized that people with greater eating disorder tendencies associate sweet foods with being overweight and, thus, participants exhibit a correlation between sweet-round matching and eating disorder tendencies. Given that drive for thinness is among the key body image concerns, our findings may support this hypothesis. The round shape in the association between sweet-round matching and drive for thinness may not be a simple geometrical shape; it may be related to body shape. This speculation is supported by the results of Experiment 2, which are related to the correlation between sweet foods, round shape, and body image concerns. Participants with greater drive for thinness associated sweet foods/round shape with obesity, weight, and dieting (Supplementary Table 2). These participants also associated sweet foods with their own bodies (Supplementary Table 2). They had stronger fears of their body shape changing as a result of eating sweet foods and stronger beliefs that they would gain weight by eating sweet foods (Supplementary Table 2). Therefore, participants with greater drive for thinness may perceive round shape as related to an overweight body shape and, thus, may be more likely to associate sweet taste with round shape.

Only drive for thinness was significantly correlated with sweet-round matching, and this result may suggest that a behavioral drive to be thinner is related to sweet-round matching. EDI2 has two subscales related to body image concerns, drive for thinness and body dissatisfaction. Body dissatisfaction is mainly assessed by questionnaire items relating to dissatisfaction with one's own body (e.g., 'I think that my hips are too big,' 'I think that my thighs are too large,' etc.) and is not associated with motivation to lose weight. On the other hand, drive for thinness includes a behavioral drive to be thinner (e.g., 'I think about going on a diet,' 'I think about losing more weight,' etc.). This behavioral drive to be thinner may cause individuals to associate sweet foods with an overweight body image, to avoid the risk of gaining weight. Obsessiveness mediation might imply that participants with greater obsessiveness believed that they should lose weight and had a stronger drive to be thinner. Given that the correlation between sweet-round matching and body mass index did not reach the statistical threshold $(r=0.122, p=0.076)$, this may imply that sweet-round matching derived from a behavioral drive to be thinner could be 
maintained after weight loss. It has been reported that some people continue their behaviors aimed at weight loss even after losing enough or excessive weight (Stice et al., 2011). And maintenance of sweet-round matching may explain this continued behavior aimed at weight loss. Thus, our results may prompt a discussion about the importance of sweet-round matching in eating disorder tendencies.

\subsection{Theoretical Contributions: the Role of Crossmodal Correspondences in Mental Disorder Tendencies}

The present study contributes new insights into the relationship between individual differences in sensory matching and mental disorder tendencies. It has been reported that individual differences in sensory matching are correlated with autistic traits in healthy people (Hidaka and Yaguchi, 2018; Yaguchi and Hidaka, 2020). The present study added a new example of the relationship between sensory matching and mental disorder tendencies by investigating eating disorder tendencies. Our mediation analysis also suggested that obsessiveness (tendency toward obsessive-compulsive disorder) is related to the formation of sensory matching. Thus, other mental disorder tendencies may be related to sensory matching. The present study may suggest the potential importance of investigating sensory matching (i.e., crossmodal correspondences) in mental disorder research fields.

\subsection{Model of Association Between Sweet-Round Matching and Drive for} Thinness

The association between taste-shape matching and drive for thinness may be explained by statistical regularities in the environment: the frequent cooccurrence of two different modal stimuli. (e.g., high pitch/small size correspondences: small/short parts in musical instruments, such as the vibraphone or the guitar, make high-pitched sounds; Spence, 2011 for a review). Participants may have had experiences such as weight gain after consuming sweet foods, being told not to eat sweet foods to avoid gaining weight, or seeing media portrayals of overweight people consuming sweet foods. Participants who reported a higher frequency of seeing/hearing information regarding the relationship between sweet foods and round body shape exhibited a higher drive for thinness (Supplementary Table 2). Previous studies investigating thoughtshape fusion (Coelho et al., 2012; Radomsky et al., 2002; Shafran et al., 1999) support the hypothesis that the statistical crossmodal correspondences model underlies our findings. These studies intensified participants' thought-shape fusion by causing them to constantly think about eating high-calorie foods, which the participants believed caused them to gain weight. Participants with greater eating disorder tendencies tend to believe more strongly that they will gain weight by simply thinking about eating high-calorie foods(Coelho et al., 
2012; Radomsky et al., 2002; Shafran et al., 1999). Thus, repeated exposure to the association between sweet foods and round body shape may have intensified the participants' beliefs that they would gain weight after eating sweet foods. Consequently, participants who had a higher drive for thinness were more likely to associate sweet taste and round shape with obesity, weight gain, and behaviors aimed at losing weight (Supplementary Table 2).

The mediating role of obsessiveness implies that obsessiveness may reinforce the association between sweet foods and round body shape. Previous studies have also reported that, after manipulation for intensifying thoughtshape fusion, people with greater obsessiveness showed stronger thoughtshape fusion (Coelho et al., 2012). The results might suggest that people with greater obsessiveness tend to associate the two repeatedly presented stimuli (i.e., sweet foods and overweight body shape) more strongly.

If obsessiveness reinforces the association between the two repeatedly presented sets of stimuli, it might suggest that the mediating role of obsessiveness could be generally applied to statistical crossmodal correspondences. From the present study, it cannot be concluded how obsessiveness reinforces the association of two sets of stimuli. However, given that the repeated co-occurrence of different modal stimuli is necessary for the establishment of statistical crossmodal correspondences, obsessiveness might cause overestimation of subjective co-occurrence experience frequency. Thus, obsessiveness facilitates the establishment of statistical crossmodal correspondences. This hypothesis may account for individual differences in the magnitude of crossmodal correspondences.

\subsection{The Role of Emotion in the Relationship Between Sweet-Round} Matching and Drive for Thinness

Aside from the role of obsessiveness, it is implied that emotional factors may mediate the correlation between sweet-round matching through the association of sweet tastes with round shapes in a unique way. The present study showed that both sweet-round matching and drive for thinness showed relationships with emotional factors. In Experiment 2, participants answered two separate questions pertaining to emotional valence (i.e., 'Do you feel positively toward sweet taste and round shape' and 'Do you feel negatively toward sweet taste and round shape'), scored on a scale ranging from 1 (strongly disagree) to 6 (strongly agree). Thus, a low score on the positive valence item does not necessarily indicate that the participant had a negative emotional response to these stimuli. Finally, participants showed a positive correlation between sweet-round matching and positive feelings toward sweet taste and round shape and positive correlation between drive for thinness and negative feelings toward sweet taste and round shape (Supplementary Table 2). It has been reported that both sweet taste and round shape have positive valence, 
and this common emotional valence guides people to associate sweet taste with round shape (Velasco et al., 2015, 2016b). Our results replicated these previous results: sweet-round matching was correlated with positive feelings toward sweet taste and round shape (Supplementary Table 2). On the other hand, there was no significant correlation between drive for thinness and positive feelings toward sweet taste and round shape. Thus, drive for thinness does not appear to influence positive feelings toward sweet taste and round shape. By contrast, a positive correlation between drive for thinness and negative feelings toward sweet taste and round shape was observed (Supplementary Table 2). Given the expression of the questionnaire items pertaining to drive for thinness (e.g., 'I am terrified of gaining weight'), drive for thinness should be classified as having negative emotional valence. Therefore, in the present context, it is suggested that drive for thinness may influence participants' negative emotional feelings toward sweet taste and round shape. It is further suggested that negative emotion could also lead to an association between sweet taste with round shape as well as a positive emotion, which is one of the present study's novel findings. Taken together, emotional factors should mediate the correlation between sweet-round matching and drive for thinness, and each positive and negative emotion may independently relate to this mediation.

Although our findings suggest that emotional mediation has a unique role, we could not perform a mediation analysis using the questionnaire data related to emotional responses to sweet taste and round shape. This was because these emotional responses correlated with either sweet-round matching or drive for thinness; correlation with both is necessary to perform mediation analysis (Supplementary Table 2). However, it should be noted that the format of our questionnaire, independently investigating positive and negative feelings toward sweet taste and round shape, may have influenced the findings. If emotional responses to sweet taste and round shape had been evaluated on a continuous scale, correlations with both sweet-round matching and drive for thinness may have been seen. This would have allowed us to analyze the mediating role of emotion in the relationship between those two factors, which should be a goal of future studies.

\subsection{Other Correlations Between Taste-Shape Matching and Eating Disorder Tendencies}

The significant correlations were not consistent between Experiments 1 and 2. In Experiment 1, positive correlations between the degree of sour-round matching and ineffectiveness, interpersonal distress, and social insecurity were observed, and the degree of bitter-round matching was negatively correlated with asceticism and perfectionism (Table 1). In Experiment 2, positive correlations between the degree of sweet-round matching and bulimia, body 
dissatisfaction, interoceptive awareness, and impulse regulation were observed (Supplementary Table 1).

Generally, sour taste is associated with angular shape (Ngo et al., 2013; Velasco et al., 2015) although the participants with greater eating disorder tendencies showed the opposite association in the present study. It was reported that patients with eating disorders showed lower sensitivity to sour tastes compared to healthy individuals (Chao et al., 2019; Kinnaird et al., 2018; for reviews). This lower sensitivity to sour taste may be due to an aberrant mental representation regarding the sour-round correspondences.

The above sensitivity hypothesis does not apply to bitter-round matching. It has also been reported that patients with eating disorders showed lower sensitivity to bitter tastes compared to healthy individuals (for reviews, see Chao et al., 2019 and Kinnaird et al., 2018). However, among our participants with greater tendencies toward eating disorders, the association between bitter taste and angular shape was stronger. Different mechanisms may relate to the negative associations of bitter-round matching with asceticism and perfectionism. Factor analysis performed in a study of a Japanese population showed that asceticism and perfectionism can be classified together (Shimura et al., 1994). Perfectionism is widely known to be strongly related to obsessive-compulsive disorder, sufferers of which show strong sensitivity to disgust stimuli (Moretz and McKay, 2008; Tolin et al., 2006). Given that bitter taste is widely recognized as being unpleasant (Ammann et al., 2019; Dubovski et al., 2017; Obrist et al., 2014), participants with higher asceticism and perfectionism may have greater sensitivity to bitter tastes, which could explain the negative correlations between bitter-round matching and asceticism and perfectionism seen herein.

Sweet-round matching correlated with other eating disorder tendencies in Experiment 2, albeit not in Experiment 1. Bulimia and body dissatisfaction are related to eating behavior and body image, as well as drive for thinness (Shimura et al., 1994). It is reported that, in Japanese healthy people, some items from drive for thinness were categorized the same factor with items from bulimia or body dissatisfaction (Shimura et al., 1994). Thus, it was speculated that these correlations may partially share a common mechanism with that relating to the correlation between sweet-round matching and drive for thinness. Interoceptive awareness and impulse regulation subscales of the EDI2 relate to self-control (Shimura et al., 1994). Thus, given that people with higher obsessiveness often show higher impulsivity (i.e., lack of self-control: Blaszczynski, 1999; Hoehn-Saric and Barksdale, 1983; Matsunaga et al., 2005), these subscales may at least partially relate to obsessiveness, which may explain the correlations with sweet-round matching.

However, we found no evidence for these suggestions, and we could not replicate these results. For now, it is speculated that these correlations were 
a Type I error. Too few participants were recruited to investigate these correlations. Therefore, future studies should investigate these correlations by recruiting larger samples.

\subsection{Limitations and Future Directions}

This study investigated the relationship between the degree of taste-shape matching and eating disorder tendency in typical, developing adults. The study has several limitations. First, and most importantly, only healthy people were recruited, and it is recommended that future studies compare differences in the degree of taste-shape matching between patients with eating disorders and healthy participants. It is hypothesized that patients with eating disorders associate sweet tastes with round shapes more than do healthy participants.

Second, the relationship between the degree of taste-shape matching and food selection and preferences was not investigated; this should also be investigated in a future study. We suspect that participants with higher sweet-round matching would tend to avoid eating sweet foods due to their higher drive for thinness.

Third, shape location (i.e., round shapes were always presented on the right-hand side) was not counterbalanced; thus, any directional preference of the participants could have affected the results. However, one-way repeated measures ANOVA showed a significant effect of taste word $\left(F_{4,210}=618\right.$, $\left.p<0.001, \eta_{p}^{2}=0.746\right)$. Moreover, a one-sample $t$-test showed that bitter $(t=$ $-14.60, p<0.001)$, salty $(t=-17.66, p<0.001)$, and sour $(t=-19.42$, $p<0.001)$ tastes were significantly skewed toward the angular end of the shape-rating scale relative to the midpoint (50), while sweet was significantly skewed toward the round end of the scale $(t=42.43, p<0.001)$. These results were similar to those presented in a previous study that counterbalanced roundness and location (Velasco et al., 2015). This suggests that any effect of direction preference would have been small in our study and, therefore, would not have a big influence on the main findings.

Fourth, actual taste stimuli were not used in our study, so we could not investigate the relationship between such taste stimuli and the shape stimuli. The decision not to use actual taste stimuli was based on previous crossmodal studies that similarly did not include actual sensory stimuli (Motoki et al., 2019b; Saluja and Stevenson, 2018; Spence et al., 2015; Velasco et al., 2015, 2018). The results of these studies suggested that crossmodal correspondences occur even when only word stimuli related to sensory perceptions are used. However, if actual taste stimuli had been used, we could have investigated a spatiotemporal mapping effect for each taste (Obrist et al., 2014). It is reported that sweet tastes provide a 'mouth filling' (lasting long time and filling the whole mouth) experience, while bitter tastes create a 'thin' (lasting long time and straight through mouth to back) experience. Spatiotemporal mapping of sweet tastes 
might be rounder and more expanded than other tastes, so that this may in turn have been associated with round body shape. Thus, individual differences in spatiotemporal mapping of sweet tastes may relate to the association between drive for thinness and sweet-round matching. This study also reported that umami conferred a similar mouth filling experience to that of sweet taste. In a future study, the role of spatiotemporal mapping in the context of sweet and umami taste stimuli should be investigated. An association of umami with round shape and a relationship between umami-round matching and drive for thinness would support a key role for spatiotemporal mapping of taste with respect to the results of the present study.

Fifth, although we could replicate the association between drive for thinness and taste-shape matching in both experiments, the effect size $(r)$ of the relationship between drive for thinness and taste-shape matching was relatively weak ( $r=0.179$ in Experiment 1 and $r=0.141$ in Experiment 2). Additionally, while the effect was significant, the indirect effect of obsessiveness was also weak $(b=0.038)$.

Sixth, the present study also encountered problems in relation to the sample size. Although we were unable to include participants who failed to respond to all of the questionnaire items in the analysis, and the final sample size was smaller than expected, power analysis nevertheless showed that a sufficient number of participants had been recruited. Based on the effect size in Experiment 1 and the number of participants in Experiment $2(n=211, r=0.179$, and $\alpha=0.05$, two-tailed), the power analysis for our main outcome measure yielded a power of 0.75 . A post-hoc power analysis combining the data from Experiments 1 and $2(n=377, r=0.157, \alpha=0.05$, two-tailed) was also performed. This post-hoc power analysis yielded a power of 0.88 , which suggests that we will probably be able to replicate the present results in a future study.

Finally, it is necessary to investigate the physiological mechanisms (e.g., perception, attention or memory function, etc.) of our findings. Earlier studies have indicated that eye-tracking or neural measures have the potential to elucidate the physiological mechanisms of food-related processing (Foerde et al., 2015; Manippa et al., 2019; Motoki et al., 2018, 2019c; Piqueras-Fiszman et al., 2013). Future studies should investigate this issue.

\subsection{Conclusions}

To summarize, our findings reveal that eating disorder tendencies are associated with the degree of taste-shape matching. Participants with a higher drive for thinness are more likely to associate sweet taste with round shape, and the relationship is mediated by obsessiveness. These findings suggest that disturbed taste-shape matching is associated with eating disorder tendencies and that obsessiveness plays a mediating role. The study's results may lead to the 
identification and implementation of effective treatment and prevention methods.

\section{Acknowledgements}

I gratefully acknowledge the financial support of the Division for Interdisciplinary Advanced Research and Education Selective Examination, Tohoku University (YH). This study was supported by KAKENHI JP17H06219 (MS), Grant-in-Aid for Research Activity start-up JP19K23384 (KM), and Grantin-Aid for JSPS Fellows JP19J21589 (YH) from the Japan Society for the Promotion of Science. The English in this document has been checked by at least two professional editors, both native speakers of English. For a certificate, please see: http://www.textcheck.com/certificate/81ADXj.

\section{Supplementary Material}

Supplementary material is available online at: https://doi.org/10.6084/m9.figshare.12661556

\section{References}

Althouse, A. D. (2016). Adjust for multiple comparisons? It's not that simple, Ann. Thorac. Surg. 101, 1644-1645. DOI:10.1016/j.athoracsur.2015.11.024.

American Psychiatric Association (2013). Diagnostic and Statistical Manual of Mental Disorders, 5th edn. American Psychiatric Association, Washington, DC, USA.

Ammann, J., Hartmann, C. and Siegrist, M. (2019). A bitter taste in the mouth: the role of 6-n-propylthiouracil taster status and sex in food disgust sensitivity, Physiol. Behav. 204, 219-223. DOI:10.1016/j.physbeh.2019.02.036.

Aspen, V., Darcy, A. M. and Lock, J. (2013). A review of attention biases in women with eating disorders, Cogn. Emot. 27, 820-838. DOI:10.1080/02699931.2012.749777.

Benjamini, Y. and Hochberg, Y. (1995). Controlling the false discovery rate: a practical and powerful approach to multiple testing, J. R. Stat. Soc. Series B Stat. Methodol. 57, 289-300. DOI:10.1111/j.2517-6161.1995.tb02031.x.

Blaszczynski, A. (1999). Pathological gambling and obsessive-compulsive spectrum disorders, Psychol. Rep. 84, 107-113. DOI:10.2466/pr0.1999.84.1.107.

Byrne, S. M., Allen, K. L., Dove, E. R., Watt, F. J. and Nathan, P. R. (2008). The reliability and validity of the dichotomous thinking in eating disorders scale, Eat. Behav. 9, 154-162. DOI:10.1016/j.eatbeh.2007.07.002.

Chao, A. M., Roy, A., Franks, A. T. and Joseph, P. V. (2019). A systematic review of taste differences among people with dating disorders, Biol. Res. Nurs. 22, 82-91. DOI:10.1177/ 1099800419872824.

Coelho, J. S., Baeyens, C., Purdon, C., Pitet, A. and Bouvard, M. (2012). Cognitive distortions and eating pathology: specificity of thought-shape fusion, Behav. Res. Ther. 50, 449-456. DOI:10.1016/j.brat.2012.04.003. 
Dazzi, F., Nitto, S. D., Zambetti, G., Loriedo, C. and Ciofalo, A. (2013). Alterations of the olfactory-gustatory functions in patients with eating disorders, Eur. Eat. Disord. Rev. 21, 382-385. DOI:10.1002/erv.2238.

Dubovski, N., Ert, E. and Niv, M. Y. (2017). Bitter mouth-rinse affects emotions, Food Qual. Pref. 60, 154-164. DOI:10.1016/j.foodqual.2017.04.007.

Efron, B. and Tibshirani, R. J. (1993). An Introduction to the Bootstrap. Chapman \& Hall/CRC, Boca Raton, FL, USA.

Ernst, M. O. (2007). Learning to integrate arbitrary signals from vision and touch, J. Vis. 7, 7. DOI:10.1167/7.5.7.

Faul, F., Erdfelder, E., Buchner, A. and Lang, A.-G. (2009). Statistical power analyses using G*Power 3.1: tests for correlation and regression analyses, Behav. Res. Methods 41, 11491160. DOI:10.3758/BRM.41.4.1149.

Feise, R. J. (2002). Do multiple outcome measures require $p$-value adjustment?, BMC Med. Res. Methodol. 2, 8. DOI:10.1186/1471-2288-2-8.

Foerde, K., Steinglass, J. E., Shohamy, D. and Walsh, B. T. (2015). Neural mechanisms supporting maladaptive food choices in anorexia nervosa, Nat. Neurosci. 18, 1571-1573. DOI:10. 1038/nn.4136.

Gardner, R. M. and Bokenkamp, E. D. (1996). The role of sensory and nonsensory factors in body size estimations of eating disorder subjects, J. Clin. Psychol. 52, 3-15. DOI:10.1002/ (SICI)1097-4679(199601)52:1<3::AID-JCLP1>3.0.CO;2-X.

Gwirtsman, H. E., Kaye, W. H., Curtis, S. R. and Lyter, L. M. (1989). Energy intake and dietary macronutrient content in women with anorexia nervosa and volunteers, J. Am. Diet. Assoc. 89, 54-57.

Hamagaki, S., Takagi, S., Urushihara, Y., Ishisaka, Y. and Matsumoto, M. (1999). Development and use of the Japanese version of the self-report Yale-Brown obsessive compulsive scale, Psychiat. Neurol. Jpn. 101, 152-168.

Hamamoto, Y., Suzuki, S., Yamazaki, S., Motoki, K., Oba, K., Kawashima, R. and Sugiura, M. (2019). Two components in body image disturbance are associated with differential neural basis, Psychother. Psychosom. 88(suppl. 1), 53. DOI:10.1159/000502467.

Hayes, A. F. (2013). Introduction to Mediation, Moderation, and Conditional Process Analysis: a Regression-Based Approach. Guilford Publications, New York, NY, USA.

Hidaka, S. and Yaguchi, A. (2018). An investigation of the relationships between autistic traits and crossmodal correspondences in typically developing adults, Multisens. Res. 31, 729 751. DOI:10.1163/22134808-20181304.

Hoehn-Saric, R. and Barksdale, V. C. (1983). Impulsiveness in obsessive-compulsive patients, Br. J. Psychiatry 143, 177-182. DOI:10.1192/bjp.143.2.177.

Jáuregui Lobera, I. and Bolaños Ríos, P. (2009). Choice of diet in patients with anorexia nervosa, Nutr. Hosp. 24, 682-687.

Kinnaird, E., Stewart, C. and Tchanturia, K. (2018). Taste sensitivity in anorexia nervosa: a systematic review, Int. J. Eat. Disord. 51, 771-784. DOI:10.1002/eat.22886.

Legenbauer, T., Vocks, S., Betz, S., Báguena Puigcerver, M. J., Benecke, A., Troje, N. F. and Rüddel, H. (2011). Differences in the nature of body image disturbances between female obese individuals with versus without a comorbid binge eating disorder: an exploratory study including static and dynamic aspects of body image, Behav. Mod. 35, 162-186. DOI:10. $1177 / 0145445510393478$. 
Li, G., Taljaard, M., Van den Heuvel, E. R., Levine, M. A. H., Cook, D. J., Wells, G. A., Devereaux, P. J. and Thabane, L. (2017). An introduction to multiplicity issues in clinical trials: the what, why, when and how, Int. J. Epidemiol. 46, 746-755. DOI:10.1093/ije/dyw320.

MacKinnon, D. P., Lockwood, C. M. and Williams, J. (2004). Confidence limits for the indirect effect: distribution of the product and resampling methods, Multivar. Behav. Res. 39, 99-128. DOI:10.1207/s15327906mbr3901_4.

Manippa, V., van der Laan, L. N., Brancucci, A. and Smeets, P. A. M. (2019). Health body priming and food choice: an eye tracking study, Food Qual. Pref. 72, 116-125. DOI:10. 1016/j.foodqual.2018.10.006.

Matsunaga, H., Kiriike, N., Matsui, T., Oya, K., Okino, K. and Stein, D. J. (2005). Impulsive disorders in Japanese adult patients with obsessive-compulsive disorder, Compr. Psychiatry 46, 43-49. DOI:10.1016/j.comppsych.2004.07.001.

Mimura, C. and Griffiths, P. (2007). A Japanese version of the Rosenberg self-esteem scale: translation and equivalence assessment, J. Psychosom. Res. 62, 589-594. DOI:10.1016/j. jpsychores.2006.11.004.

Mohr, H. M., Zimmermann, J., Röder, C., Lenz, C., Overbeck, G. and Grabhorn, R. (2010). Separating two components of body image in anorexia nervosa using fMRI, Psychol. Med. 40, 1519-1529. DOI:10.1017/S0033291709991826.

Moretz, M. W. and McKay, D. (2008). Disgust sensitivity as a predictor of obsessivecompulsive contamination symptoms and associated cognitions, J. Anxiety Disord. 22, $707-$ 715. DOI:10.1016/j.janxdis.2007.07.004.

Motoki, K., Saito, T., Nouchi, R., Kawashima, R. and Sugiura, M. (2018). Tastiness but not healthfulness captures automatic visual attention: preliminary evidence from an eye-tracking study, Food Qual. Pref. 64, 148-153. DOI:10.1016/j.foodqual.2017.09.014.

Motoki, K., Saito, T., Nouchi, R., Kawashima, R. and Sugiura, M. (2019a). Round faces are associated with sweet foods: the role of crossmodal correspondence in social perception, Foods 8, 103. DOI: $10.3390 /$ foods8030103.

Motoki, K., Saito, T., Nouchi, R. and Kawashima, R. (2019b). Light colors and comfortable warmth: crossmodal correspondences between thermal sensations and color lightness influence consumer behavior, Food Qual. Pref. 72, 45-55. DOI:10.1016/j.foodqual.2018.09.004.

Motoki, K., Saito, T., Nouchi, R., Kawashima, R. and Sugiura, M. (2019c). Anxiety increases visual attention to hedonic foods: a preliminary eye-tracking study on the impact of the interplay between integral and incidental affect on foods, Appetite 137, 218-225. DOI:10. 1016/j.appet.2019.02.009.

Motoki, K., Saito, T., Park, J., Velasco, C., Spence, C. and Sugiura, M. (2020). Tasting names: systematic investigations of taste-speech sounds associations, Food Qual. Pref. 80, 103801. DOI:10.1016/j.foodqual.2019.103801.

Ngo, M. K., Velasco, C., Salgado, A., Boehm, E., O’Neill, D. and Spence, C. (2013). Assessing crossmodal correspondences in exotic fruit juices: the case of shape and sound symbolism, Food Qual. Pref. 28, 361-369. DOI:10.1016/j.foodqual.2012.10.004.

Oberndorfer, T. A., Frank, G. K. W., Simmons, A. N., Wagner, A., McCurdy, D., Fudge, J. L., Yang, T. T., Paulus, M. P. and Kaye, W. H. (2013). Altered insula response to sweet taste processing after recovery from anorexia and bulimia nervosa, Am. J. Psychiatry 170, 11431151. DOI:10.1176/appi.ajp.2013.11111745. 
Obrist, M., Comber, R., Subramanian, S., Piqueras-Fiszman, B., Velasco, C. and Spence, C. (2014). Temporal, affective, and embodied characteristics of taste experiences: a framework for design, in: CHI'14: Proceedings of the SIGCHI Conference on Human Factors in Computing Systems, pp. 2853-2862. DOI:10.1145/2556288.2557007.

Oshio, A. (2009). Development and validation of the dichotomous thinking inventory, Soc. Behav. Personal. 37, 729-742. DOI:10.2224/sbp.2009.37.6.729.

Oshio, A. and Meshkova, T. (2012). Eating disorders, body image, and dichotomous thinking among Japanese and Russian college women, Health 4, 392-399. DOI:10.4236/health.2012. 47062.

Palascha, A., van Kleef, E. and van Trijp, H. C. M. (2015). How does thinking in black and white terms relate to eating behavior and weight regain?, J. Health Psychol. 20, 638-648. DOI:10.1177/1359105315573440.

Parise, C. V., Knorre, K. and Ernst, M. O. (2014). Natural auditory scene statistics shapes human spatial hearing, Proc. Natl Acad. Sci. USA 111, 6104-6108. DOI:10.1073/pnas.1322705111.

Piqueras-Fiszman, B., Velasco, C., Salgado-Montejo, A. and Spence, C. (2013). Using combined eye tracking and word association in order to assess novel packaging solutions: a case study involving jam jars, Food Qual. Pref. 28, 328-338. DOI:10.1016/j.foodqual.2012.10. 006.

Preacher, K. J. and Hayes, A. F. (2004). SPSS and SAS procedures for estimating indirect effects in simple mediation models, Behav. Res. Methods Instrum. Comput. 36, 717-731. DOI:10. 3758/BF03206553.

Pritchard, T. C., Macaluso, D. A. and Eslinger, P. J., et al. (1999). Taste perception in patients with insular cortex lesions, Behav. Neurosci. 113, 663-671. DOI:10.1037/0735-7044.113.4. 663.

Radomsky, A. S., de Silva, P., Todd, G., Treasure, J. and Murphy, T. (2002). Thought-shape fusion in anorexia nervosa: an experimental investigation, Behav. Res. Ther. 40, 1169-1177. DOI:10.1016/S0005-7967(01)00085-7.

Saluja, S. and Stevenson, R. J. (2018). Cross-modal associations between real tastes and colors, Chem. Senses 43, 475-480. DOI:10.1093/chemse/bjy033.

Schoemaker, C., van Strien, T. and van der Staak, C. (1994). Validation of the eating disorders inventory in a nonclinical population using transformed and untransformed responses, Int. J. Eat. Disord. 15, 387-393. DOI:10.1002/eat.2260150409.

Shafran, R., Teachman, B. A., Kerry, S. and Rachman, S. (1999). A cognitive distortion associated with eating disorders: thought-shape fusion, Br. J. Clin. Psychol. 38, 167-179. DOI:10. 1348/014466599162728.

Shimura, M., Horie, H., Kumano, H., Kuboki, T., Suematsu, H. and Sakano, Y. (1994). Factor structure analysis of the Japanese version of the eating disorder inventory-91, Jpn. Assoc. Behav. Cogn. Ther. 20, 8-15.

Smeets, M. A. M., Ingleby, J. D., Hoek, H. W. and Panhuysen, G. E. M. (1999). Body size perception in anorexia nervosa: a signal detection approach, J. Psychosom. Res. 46, 465477. DOI:10.1016/S0022-3999(99)00005-7.

Spence, C. (2011). Crossmodal correspondences: a tutorial review, Atten. Percept. Psychophys. 73, 971-995. DOI:10.3758/s13414-010-0073-7. 
Spence, C. (2019). Assessing the role of emotional mediation in explaining crossmodal correspondences involving musical stimuli, Multisens. Res. 33, 1-29. DOI:10.1163/2213480820191469.

Spence, C. and Deroy, O. (2013). On the shapes of flavours: a review of four hypotheses, Theor. Hist. Sci. 10, 207-238. DOI:10.12775/ths-2013-0011.

Spence, C., Wan, X., Woods, A., Velasco, C., Deng, J., Youssef, J. and Deroy, O. (2015). On tasty colours and colourful tastes? Assessing, explaining, and utilizing crossmodal correspondences between colours and basic tastes, Flavour 4, 23. DOI:10.1186/s13411-0150033-1.

Stice, E., Marti, C. N. and Durant, S. (2011). Risk factors for onset of eating disorders: evidence of multiple risk pathways from an 8-year prospective study, Behav. Res. Ther. 49, 622-627. DOI:10.1016/j.brat.2011.06.009.

Tolin, D. F., Woods, C. M. and Abramowitz, J. S. (2006). Disgust sensitivity and obsessivecompulsive symptoms in a non-clinical sample, J. Behav. Ther. Exp. Psychiatry 37, 30-40. DOI:10.1016/j.jbtep.2005.09.003.

Turoman, N., Velasco, C., Chen, Y.-C., Huang, P.-C. and Spence, C. (2018). Symmetry and its role in the crossmodal correspondence between shape and taste, Atten. Percept. Psychophys. 80, 738-751. DOI: 10.3758/s13414-017-1463-x.

Velasco, C., Woods, A. T., Deroy, O. and Spence, C. (2015). Hedonic mediation of the crossmodal correspondence between taste and shape, Food Qual. Pref. 41, 151-158. DOI:10. 1016/j.foodqual.2014.11.010.

Velasco, C., Woods, A. T., Petit, O., Cheok, A. D. and Spence, C. (2016a). Crossmodal correspondences between taste and shape, and their implications for product packaging: a review, Food Qual. Pref. 52, 17-26. DOI:10.1016/j.foodqual.2016.03.005.

Velasco, C., Woods, A. T., Marks, L. E., Cheok, A. D. and Spence, C. (2016b). The semantic basis of taste-shape associations, PeerJ 4, e1644. DOI:10.7717/peerj.1644.

Velasco, C., Hyndman, S. and Spence, C. (2018). The role of typeface curvilinearity on taste expectations and perception, Int. J. Gastron. Food Sci. 11, 63-74. DOI:10.1016/j.ijgfs.2017. 11.007.

Wagner, A., Simmons, A. N., Oberndorfer, T. A., Frank, G. K. W., McCurdy-McKinnon, D., Fudge, J. L., Yang, T. T., Paulus, M. P. and Kaye, W. H. (2015). Altered sensitization patterns to sweet food stimuli in patients recovered from anorexia and bulimia nervosa, Psychiatry Res. Neuroimaging 234, 305-313. DOI:10.1016/j.pscychresns.2015.10.010.

Yaguchi, A. and Hidaka, S. (2020). Unique relationships between autistic traits and visual, auditory, and tactile sensory thresholds in typically developing adults, Perception 49, 405421. DOI:10.1177/0301006620907827. 